\title{
BMJ Open Scoping review protocol: education initiatives for medical psychiatry collaborative care
}

\author{
Nelson Shen, ${ }^{1}$ Sanjeev Sockalingam, ${ }^{2,3}$ Alexxa Abi Jaoude, ${ }^{4}$ Sharon M Bailey, ${ }^{4}$ \\ Thérèse Bernier, ${ }^{1}$ Alison Freeland, ${ }^{2,5}$ Aceel Hawa, ${ }^{4}$ Elisa Hollenberg, ${ }^{4}$ \\ Bethel Woldemichael, ${ }^{4}$ David Wiljer ${ }^{1,2,3,5,6}$
}

To cite: Shen N, Sockalingam S, Abi Jaoude A, et al. Scoping review protocol: education initiatives for medical psychiatry collaborative care. BMJ Open 2017;7:e015886. doi:10.1136/ bmjopen-2017-015886

- Additional File 1. Search Strategy.

- Prepublication history and additional material for this paper are available online. To view please visit the journal (http:// dx.doi.org/10.1136/bmjopen2017-015886).

Received 9 January 2017 Revised 20 July 2017

Accepted 21 July 2017

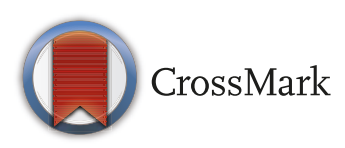

${ }^{1}$ Institute of Health Policy, Management and Evaluation, University of Toronto, Toronto, Ontario, Canada

${ }^{2}$ Department of Psychiatry, University of Toronto, Toronto,

Ontario, Canada

${ }^{3}$ Centre for Addiction and Mental Health, University Health Network, Toronto, Ontario, Canada

${ }^{4}$ Department of Education, Centre for Addiction and Mental Health, Toronto, Ontario, Canada ${ }^{5}$ Trillium Health Partners, Mississauga, Ontario, Canada ${ }^{6}$ UHN Digital, University Health Network, Toronto, Ontario, Canada

Correspondence to Dr David Wiljer; david.wiljer@uhn.ca

\section{ABSTRACT}

Introduction The collaborative care model is an approach providing care to those with mental health and addictions disorders in the primary care setting. There is a robust evidence base demonstrating its clinical and costeffectiveness in comparison with usual care; however, the transitioning to this new paradigm of care has been difficult. While there are efforts to train and prepare healthcare professionals, not much is known about the current state of collaborative care training programmes. The objective of this scoping review is to understand how widespread these collaborative care education initiatives are, how they are implemented and their impacts. Methods and analysis The scoping review methodology uses the established review methodology by Arksey and 0'Malley. The search strategy was developed by a medical librarian and will be applied in eight different databases spanning multiple disciplines. A two-stage screening process consisting of a title and abstract scan and a full-text review will be used to determine the eligibility of articles. To be included, articles must report on an existing collaborative care education initiative for healthcare providers. All articles will be independently assessed for eligibility by pairs of reviewers, and all eligible articles will be abstracted and charted in duplicate using a standardised form. The extracted data will undergo a 'narrative review' or a descriptive analysis of the contextual or process-oriented data and simple quantitative analysis using descriptive statistics.

Ethics and dissemination Research ethics approval is not required for this scoping review. The results of this scoping review will inform the development of a collaborative care training initiative emerging from the Medical Psychiatry Alliance, a four-institution philanthropic partnership in Ontario, Canada. The results will also be presented at relevant national and international conferences and published in a peer-reviewed journal.

\section{BACKGROUND}

There is a growing disparity of unmet needs for mental health services ${ }^{12}$ as the current supply of psychiatrists and current practice patterns create substantial barriers hindering access to psychiatric assessment and treatment. ${ }^{3-6}$ In recent years, primary

\section{Strengths and limitations of this study}

- The results of this review will establish a baseline understanding of the delivery of education initiatives for collaborative care-a timely and important topic required to support the transition to a more integrated delivery of mental health and addiction care.

- This protocol outlines a rigorous study design that includes the use of an established scoping review methodology, a multidisciplinary search strategy developed iteratively in consultation with an experienced medical librarian and a study selection and data extraction process that is carried out in tandem with validation from content experts.

- A limitation of the review is the potential to miss relevant articles given that education is not always separated from the implementation of collaborative care; however, the reference lists of included articles, relevant literature reviews and key reports will be hand-searched to identify articles missed by the search strategy.

- No formal quality assessment will be conducted as this review aims to provide a snapshot of the landscape of collaborative care education initiatives by being inclusive of all types of information available.

- While the review will be non-discriminant towards article types and methodologies, the findings will be limited to articles written in English.

care has increasingly become the first contact point for those seeking mental health treatment ${ }^{7}$; however, individuals seeking care in these settings often receive suboptimal care as providers are not properly equipped or trained to manage complex physical and mental health conditions. ${ }^{9}$ The disconnect with mental health services contributes to the treatment gap, making it difficult for individuals to receive the proper care, especially for those with comorbidity or multimorbidity. This inattention may further exacerbate their condition or lead to premature death due to their physical health conditions. ${ }^{10-12}$ 
Backed by a growing and robust evidence base, there is a case for integrating mental healthcare into the primary care setting through a collaborative care model. Several meta-analyses ${ }^{13-17}$ have demonstrated that collaborative care models can be more effective in treating mental health disorders than usual care. The collaborative care model has also demonstrated its value by improving quality of life for comorbid patients for no or modest additional cost. ${ }^{18}$

Collaborative care is often used interchangeably with other terms (eg, mental health integration, integrated care and integrated mental health) to describe a range of models of care that consist of healthcare professionals working in partnership in a primary care setting to deliver mental healthcare; however, the degree of integration of the two disciplines vary depending on model. ${ }^{19}$ Recently, an American Psychiatric Association/American Psychosomatic Medicine (APA/APM) working group to provide clarity and a standardised evidence-based integrated care model. Derived from the seminal research by Katon et $a l^{20}$ and Wagner's Chronic Care Model, ${ }^{21}$ the APA/ APM Collaborative Care Model $^{22}$ defines collaborative care as the provision of care that is: (1) team driven, (2) population focused, (3) measurement guided and (4) evidence based. The focal point is the collaborative care team that consists of a multidisciplinary group of professionals (eg, psychiatric nurse practitioner, social worker, licenced counsellor or therapist, psychologist or psychiatrist, care managers and office support staff) thereby extending beyond the 'physician as treatment team' in providing and supporting care and implementing and revising the treatment plan. The new definition was developed to support informed decision making by policymakers, healthcare providers, service delivery organisations and the public and to help standardise future training in this area.

Despite the increasing recognition and implementation of the collaborative care model, there remain difficulties in implementation as there is a lack of adequately prepared workforce. ${ }^{23}$ The transition from a traditional care delivery model is a challenge as there are many new processes and systems-level differences between integrated behavioural health and traditional primary care settings that new team members must navigate. ${ }^{24} 25$ Many programmes have been developed and implemented to provide training to provide individuals with the necessary skills, knowledge, competencies and attitudes to support the collaborative model of care. ${ }^{23-25}$ A variety of training approaches have been implemented, including training by internal or external experts, onsite or offsite sessions, onboarding processes for new employees, training manuals, shadowing processes and peer mentoring. ${ }^{23} 25$ However, there have been calls for increased prelicensure training to take the burden off of such workplace training programmes that can be expensive and resource intensive. ${ }^{23}$ Furthermore, a recent editorial ${ }^{26}$ has identified the need to move beyond competency-based training in integrated care and called for integration of theoretical frameworks, namely adaptive expertise, to better prepare future healthcare professionals for managing complexity within integrated care settings.

While there have been tremendous efforts in the development of the collaborative care model and the training supports, not much is known about the current scope of integrated care education, how it is delivered, to whom, in what contexts, the content of the training and whether it is effective. There is a need to collect all the knowledge and experiences from existing training programmes, to continually improve current process of these programmes and to support the development of new programmes. The objective of this scoping review is to gain an understanding of the current landscape of integrated care education.

\section{METHODS}

The purpose of this review is to gain an understanding of the extent to which collaborative care education programs have been implemented and the factors which contributed or detered to program success. The findings from this review will be used by the Medical Psychiatry Alliance - a four-institution, Ministry of Health and Long-Term Care, and philanthropic partnership in Ontario, Canada - to inform the development of thier collaborative care training initiative. Various knowledge synthesis approaches were considered for this review; however, the scoping review methodology is most appropriate especially since the complex area of collaborative care education has not been reviewed comprehensively before. ${ }^{27} 28$ To the authors' knowledge, there has been no prior attempt to establish a baseline of knowledge regarding collaborative care education initiatives. Given this knowledge gap and that literature may be diffuse due to the multidisciplinary nature of collaborative care, scoping reviews are ideal in taking stock of the volume and nature of the literature. ${ }^{28}$ Using this form of knowledge synthesis allows for the broad exploration of collaborative care education to map key concepts, evidence types and gaps in research in a defined field; furthermore, scoping review makes use of a wide array of knowledge exhibited through empirical research and anecdotal accounts. ${ }^{29-31}$

The methodology for this review draws on Arksey and O'Malley's seminal framework ${ }^{29}$ for scoping reviews as the foundation and more recent advancements to the methodology. ${ }^{30}{ }^{32}$ As recommended by Colquhoun et $a l^{33}$ this protocol follows the relevant aspects of the Preferred Reporting Items for Systematic Review and Meta-Analysis Protocols (PRISMA-P) guidelines ${ }^{34}$ to ensure rigour in reporting the methodology in the interim, while PRISMA guidelines are developed for scoping reviews. ${ }^{35}$ Scoping reviews share a similar process as systematic reviews since they both are rigorous and transparent in identifying eligible literature but are divergent in purpose as scoping reviews aim to map the body of literature rather than sum up the best available research on a specific question. ${ }^{36}$ Scoping reviews are often seen as a precursor to 
systematic reviews as it allows researchers to determine the value and probable scope of a full systematic review and meta-analysis. ${ }^{29} 3738$

\section{Stage 1: identifying the research question}

The objective of this review is to understand the current state of collaborative care education initiatives by identifying existing initiatives (eg, training programmes and interventions) reported in both academic and grey literature. By identifying the past and current education initiatives, this review seeks to establish a foundational understanding of how these programmes were implemented and glean the critical success factors and recommendations of these experiences. To meet these objectives, this review asks the following questions:

- What educational interventions exist within integrated collaborative care programmes in hospital, community and primary care settings?

- What aspects of collaborative care are taught in the educational interventions?

- How well do the interventions incorporate the core collaborative care principles as outlined in the APA/APM document?

- How were the interventions delivered? What were the critical success factors and lessons learnt?

While the primary focus of this review is to take an inventory of existing programmes and their processes, this review will also provide a narrative view on the extent to which these initiatives have been evaluated and provide a descriptive review of the effectiveness of current efforts in educating the health professions about collaborative care. The review will ask 'What aspects of collaborative care programs have been evaluated' and 'What were the outcomes of the evaluations (eg, user perceptions, attitudinal changes, changes in knowledge and competency, behaviour change, organisational and clinical impact)?'

\section{Stage 2: identifying relevant studies}

The search strategy was iteratively developed by the research team in collaboration with an experienced medical librarian (SB) and implemented on July 2016 in eight electronic databases: Medline, Medline In-Process, PsycINFO, Embase, CINAHL, ERIC, Scopus and ISI Web of Science. These databases were selected to capture a comprehensive sample of literature from health sciences, psychiatry, education and other disciplines. The search query was first developed for Medline. Medline (Ovid) was selected as the first database to query because the Ovid interface facilitates fine-tuning at a level that PubMed does not; moreover, an added advantage to using Medline is its use of the National Library of Medicine's controlled vocabulary, MeSH, to index citations. ${ }^{39}$ Any chance in missing articles from PubMed were reduced by searching Ovid Medline 'In-Process \& Other Non-Indexed Citation' database to capture the most recent literature possible. The Ovid interface is also a shared platform that allows for quicker translation and querying of other Ovid-based databases (Medline In-Process, PsycINFO and Embase).
The search strategy consisted of subject headings, keywords and related terms for primary care, integrated care, education and mental health services, personnel and conditions. Depending on the database, some subject terms were 'exploded', which allowed us to capture all relevant search topics under a given term (eg, using 'exp mental disorders/' in Medline will catch all possible mental health diagnoses and conditions). Terms and concepts were combined using Boolean logic and operators including adjacencies. The searches were limited to articles in English and published after 1995-when the collaborative care model was first introduced. ${ }^{20} 22$ The search terms were then translated for use in the other databases. Applying the same search string to ISI Web of Science (interdisciplinary) required some modifications and a different approach to reduce the noise in the results. Specifically, the research categories and subject area limiters were used to reduce the yield to a manageable volume while maintaining the specificity required for this review. The first 100 search results from each database were reviewed by the research team to ensure validity of the search strategy (see online additional file 1 for full strategy).

The results from the search were imported into Mendeley desktop reference manager where the citations were collated and deduplicated. The research team was granted access to the citations and articles using the Mendeley web-based collaboration function. The citations were then copied and pasted into a spreadsheet for use in the subsequent eligibility screening and charting processes.

\section{Stage 3: study selection}

A two-stage screening process consisting of a title and abstract scan and a full-text review will be used to determine the eligibility of articles. Both stages will follow the same process, where every article will be independently reviewed in pairs and the results will be documented on the spreadsheet. At the end of each round, the ratings will be compared and resolved by the two reviewers or a third reviewer when consensus is not achieved. Any ambiguities regarding the eligibility of a citation (or article) will be flagged and discussed.

The citations will be assessed for relevance based on a title and abstract scan. To be relevant for full-text review, the title and abstract must: focus on providers from different specialities, disciplines or sectors working together to offer complementary services or support in delivering care; be about delivering mental health and addictions care; and describe an existing education intervention. This review is inclusive of all types of literature, thus including commentary articles, case studies and empirical studies employing all types of methodologies (ie, qualitative, quantitative and mixed methods) and study designs. Viewpoint articles on how education programmes should be implemented outside of the context of an existing programme are excluded. 
The criteria will be piloted by the reviewers to refine and establish a common understanding of the inclusion criteria. After a training session, $20 \%$ of the Medline citations will be independently reviewed by four reviewers to establish inter-rater reliability (IRR). The results of the review will be compared, and the IRR will be calculated. The threshold for IRR is set at an average Cohen's K of 0.70 indicating substantial agreement. ${ }^{40}$ The pilot will be run again if the threshold was not met. If met, the remaining articles will then be divided and assigned to two sets of pairs for independent review. These adjustments to the inclusion-exclusion process are appropriate as they provide the team with opportunities to become familiar with the data and to reduce workload. ${ }^{30} 32$ This protocol made an additional adjustment by adding the IRR to establish agreement between reviewers and provide more context to the study selection process. Regardless of the IRR outcome, a meeting about the process will be held to compare the results, resolve the disagreements and troubleshoot the challenges that arose during the titleabstract review process.

Relevant articles identified in the title and abstract scan will undergo a full-text review to confirm the article's eligibility for the review. The full-text review follows the same process as the title and abstract scan. To be included, the article must be about a collaborative care education initiative for healthcare providers. The full-text review form asked reviewers to assess each article using the following questions:

1. Does the article describe/discuss the provision of care related to mental health in a primary care setting?

2. Does the article describe an education intervention/ programme?

3. Does the education intervention focus on delivering team-based care?

\section{Step 4: charting the data}

A standardised charting form will be developed by the research team to allow the investigators to categorise or 'chart' the data. The high-level domains for the charting form consist of article details, study details (if applicable), initiative details and implementation factors. The specifics of each domain are outlined in table 1 . There will be a training session to trial the charting form and ensure there is a common understanding of the categories and how to use the form. The full-text reviewers will be asked if there are any additional variables emerging from the full-text review to consider for charting. The form will be piloted on 5-10 articles by the team. This will consist of independent charting by the reviewers and validation by the senior investigators. A final round of feedback on the form will be solicited prior to the charting process. The charting will also consist of independent charting by the reviewers and validation by the senior investigators. The charters will be encouraged to provide constant feedback on emerging themes not captured in the charting form. The form will be revised as required.

\section{Stage 5: collating, summarising and reporting the results}

The extracted data will first undergo a simple quantitative analysis using descriptive statistics (eg, frequencies and central tendency measures) to provide numerical summaries of the education initiatives and article or study characteristics. ${ }^{29}$ Multiple articles stemming from a single initiative will be grouped and treated as a unit of analysis. The data will also undergo a 'narrative review' or a descriptive analysis of the contextual or process-oriented data where all data will be thematically analysed independently by two reviewers to identify emerging themes found within each of the subdomains outlined in table 1 . The results will be compared and consolidated by consensus between the two reviewers. The resulting themes will be reviewed by content experts to ensure validity and credibility. The themes will be reported to highlight the similarities, patterns, differences and outliers found in the literature.

The results from empirical studies (ie, qualitative, quantitative and mixed methods) will be classified into learner and clinical outcomes based on the Kirkpatrick-Barr framework ${ }^{41}$ for interprofessional learner outcomes. This framework was selected because of its focus on interprofessional collaboration that can be applicable to the multidisciplinary setting. Thematic analysis will also be used to identify commonalities within each of the levels of the following outcome typology:

- Level 1: learners' reaction-participant views of the learning experience and satisfaction with the programme;

- Level 2a: modification of attitudes/perceptionschanges in reciprocal attitudes or perceptions between participant groups, towards patients/ clients and their condition, circumstances, care and treatment;

- Level 2b: acquisition of knowledge/skills—changes in knowledge and skills;

- Level 3: change in behaviour-changes in behaviour transferred from the learning environment to the workplace;

- Level 4a: change in organisational practice-changes in the organisation or delivery of care attributable to an education programme;

- Level 4b: benefits to patients/clients-improvements in the health and well-being of patients/clients as a direct result of an education programme.

Details of the education initiatives and study outcomes will be summarised in a table. The articles will not be assessed for quality as it is outside the scope of this review; however, details of the included articles (ie, article type and methodology) will be reported in a summary table to provide context of the maturity of the evidence. 
Table 1 Data charting domains and elaboration of subdomains

\section{Domain/} subdomains

Article details Article type

Year Country Study details (if applicable)

Study design

Participants Intervention

Comparator

Study outcomes

Outcomes

Initiative details

\begin{tabular}{|c|c|}
\hline Name & What is the name of the programme (if applicable)? \\
\hline Setting & $\begin{array}{l}\text { Where does the education programme take place (eg, community, hospital and } \\
\text { university)? }\end{array}$ \\
\hline Programme delivery & How is the programme delivered (eg, seminar, lecture, course and in-service training)? \\
\hline Instructors & Who are the facilitators/instructors? \\
\hline 1. Team Driven & $\begin{array}{l}\text { Does the programme teach a team-based approach of multiprofessionals to provide } \\
\text { and support care and monitor treatment plans? }\end{array}$ \\
\hline 2. Specific population & $\begin{array}{l}\text { Does the programme focus on the provision of care and health outcomes of a defined } \\
\text { population of patients? }\end{array}$ \\
\hline \multicolumn{2}{|l|}{ Implementation factors } \\
\hline Success & How did the authors define programme success? \\
\hline Enablers & What factors that contributed to the success of the programme? \\
\hline Barriers & What factors may have detracted from the success of the programme? \\
\hline Recommendations & What were the author's recommendations based on their experiences? \\
\hline
\end{tabular}

APA/APM, American Psychiatric Association/American Psychosomatic Medicine.

\section{ETHICS/DISSEMINATION}

This protocol reports a comprehensive, rigorous and transparent methodology. This review contributes of the advancement of research on this subject and comment on the maturity of the body of literature by identifying gaps in knowledge and research. Through the publication of the results and dissemination at relevant conferences, the results of this review could guide the direction of future research. The results from this review may inform the design of new initiatives and the policies that support them; moreover, future implementations can learn from the experience of others to avoid potential barriers and focus on enablers to increase the chances of success of their programmes-existing or new.

Acknowledgements The authors' work is supported in part by the Medical Psychiatry Alliance, a collaborative health partnership of the University of Toronto, the Centre for Addiction and Mental Health, the Hospital for Sick Children, Trillium Health Partners, the Ontario Ministry of Health and Long-Term Care and an anonymous donor. The authors would also like to thank Rebecca Charow for her fresh eyes in proofreading and editing the manuscript.

Contributors NS led the design and conceptualisation of this work and drafted the protocol. AAJ, TB, AH, EH and BW were involved in the conceptualisation of the review design, specifically in establishing the inclusion and exclusion criteria. $\mathrm{EH}$ and BW drafted the background section of the protocol and provided feedback on the methodology and the manuscript. SMB developed the search strategy, conducted the search, provided feedback on the manuscript and copy-edited the manuscript. AF, SS and DW provided guidance to the conceptualisation and design 
of the study and data analyses and have revised all drafts of this manuscript for important intellectual content and clarity. All authors give approval to the publishing of this protocol manuscript.

\section{Competing interests None declared.}

Provenance and peer review Not commissioned; externally peer reviewed.

Open Access This is an Open Access article distributed in accordance with the Creative Commons Attribution Non Commercial (CC BY-NC 4.0) license, which permits others to distribute, remix, adapt, build upon this work non-commercially, and license their derivative works on different terms, provided the original work is properly cited and the use is non-commercial. See: http://creativecommons.org/ licenses/by-nc/4.0/

(c) Article author(s) (or their employer(s) unless otherwise stated in the text of the article) 2017. All rights reserved. No commercial use is permitted unless otherwise expressly granted.

\section{REFERENCES}

1. Roll JM, Kennedy J, Tran M, et al. Disparities in unmet need for mental health services in the United States, 1997-2010. Psychiatr Serv 2013;64:80-2.

2. Sunderland A, Findlay LC. Perceived need for mental health care in Canada: Results from the 2012 Canadian community health surveymental health. Health Rep 2013;24:3-9.

3. Goldner EM, Jones W, Fang ML. Access to and waiting time for psychiatrist services in a Canadian urban area: a study in real time. Can J Psychiatry 2011;56:474-80.

4. Bishop TF, Press MJ, Keyhani S, et al. Acceptance of insurance by psychiatrists and the implications for access to mental health care. JAMA Psychiatry 2014;71:176-81.

5. Kurdyak P, Zaheer J, Cheng J, et al. Changes in characteristics and practice patterns of Ontario Psychiatrists. Can J Psychiatry 2017;62.

6. Cunningham PJ. Beyond parity: primary care physicians' perspectives on access to mental health care. Health Aff 2009;28:w490-w501.

7. Wang PS, Lane M, Olfson M, et al. Twelve-month use of mental health services in the United States. Arch Gen Psychiatry 2005;62:629.

8. Wang PS, Demler O, Olfson M, et al. Changing profiles of service sectors used for mental health care in the United States. Am J Psychiatry 2006;163:1187-98.

9. Knickman J, Krishnan R, Pincus H. Improving access to effective care for people with mental health and substance use disorders. JAMA 2016;316:1647.

10. Ivbijaro GO, Enum $\mathrm{Y}$, Khan AA, et al. Collaborative care: models for treatment of patients with complex medical-psychiatric conditions. Curr Psychiatry Rep 2014;16:506.

11. Patel V, Belkin GS, Chockalingam A, et al. Grand challenges: integrating mental health services into priority health care platforms. PLoS Med 2013;10:e1001448.

12. Walker ER, McGee RE, Druss BG. Mortality in mental disorders and global disease burden implications. JAMA Psychiatry 2015;72:334-41.

13. Gilbody S. Collaborative Care for Depression. Arch Intern Med 2006;166:2314.

14. Thota AB, Sipe TA, Byard GJ, et al. Collaborative care to improve the management of depressive disorders: a community guide systematic review and meta-analysis. Am J Prev Med 2012;42:525-38.

15. Archer J, Bower P, Gilbody S, et al. Collaborative care for depression and anxiety problems. Cochrane Database Syst Rev 2012;10:CD006525.

16. Coventry PA, Hudson JL, Kontopantelis E, et al. Characteristics of effective collaborative care for treatment of depression: a systematic review and meta-regression of 74 randomised controlled trials. PLoS One 2014;9:e108114.
17. Panagioti M, Bower P, Kontopantelis E, et al. Association between chronic physical conditions and the effectiveness of collaborative care for depression. JAMA Psychiatry 2016;73:978.

18. Katon W, Russo J, Lin EH, Ehb L, et al. Cost-effectiveness of a multicondition collaborative care intervention: a randomized controlled trial. Arch Gen Psychiatry 2012;69:506-14.

19. Kwan BM, Nease DE. The State of the Evidence for Integrated Behavioral Health in Primary Care. In: Integrated Behavioral Health in Primary Care. New York, NY: Springer New York, 2013:65-98.

20. Katon W. Collaborative care: Patient satisfaction, outcomes, and medical cost-offset. Fam Syst Med 1995;13:351-65.

21. Wagner EH. Meeting the needs of chronically ill people. BMJ 2001;323:945-6.

22. American Psychiatric Association, Academy of Psychosomatic Medicine. Dissemination of Integrated Care Within Adult Primary Care Settings: The Collaborative Care Model, 2016.

23. Hall J, Cohen DJ, Davis M, et al. Preparing the workforce for behavioral health and primary care integration. J Am Board Fam Med 2015;28(Suppl 1):S41-51.

24. Ratzliff A, Norfleet K, Chan YF, et al. Perceived educational needs of the integrated care psychiatric consultant. Acad Psychiatry 2015;39:448-56.

25. Dickinson WP. Strategies to support the integration of behavioral health and primary care: what have we learned thus far? J Am Board Fam Med 2015;28(Suppl 1):S102-6.

26. Sockalingam S, Mulsant BH, Mylopoulos M. Beyond integrated care competencies: the imperative for adaptive expertise. Gen Hosp Psychiatry 2016;43:30-1.

27. Mays N, Roberts E, Popay J. Synthesising research evidence. In. Studying the organisation and delivery of health services: Research methods, 2001:188-220.

28. Wilson MG, Lavis JN, Guta A. Community-based organizations in the health sector: a scoping review. Health Res Policy Syst 2012;10:36.

29. Arksey H, O'Malley L. Scoping studies: towards a methodological framework. Int J Soc Res Methodol 2005;8:19-32.

30. Levac D, Colquhoun H, O'Brien KK. Scoping studies: advancing the methodology. Implement Sci 2010;5:69.

31. Colquhoun HL, Levac D, O'Brien KK, et al. Scoping reviews: time for clarity in definition, methods, and reporting. J Clin Epidemiol 2014;67:1291-4.

32. Daudt HM, van Mossel C, Scott SJ. Enhancing the scoping study methodology: a large, inter-professional team's experience with Arksey and O'Malley's framework. BMC Med Res Methodol 2013;13:48

33. Colquhoun H. Current Best Practices for the Conduct of Scoping Reviews. EQUATOR Netw 2016. http://www.equator-network.org/ wp-content/uploads/2016/06/Gerstein-Library-scoping-reviews May-12.pdf (accessed 20 May 2017).

34. Shamseer L, Moher D, Clarke M, et al. Preferred reporting items for systematic review and meta-analysis protocols (PRISMA-P) 2015: elaboration and explanation. BMJ 2015;349:g7647-g7647.

35. Tricco A, Straus S, Moher D. Preferred reporting items for systematic reviews and meta-analysis: extension for Scoping Reviews (PRISMA$\mathrm{ScR}$ ). EQUATOR Netw http://www.equator-network.org/wp-content/ uploads/2009/02/Executive-summary_ScR_Dec-9.pdf (accessed 20 May 2017).

36. Pham MT, Rajić A, Greig JD, et al. A scoping review of scoping reviews: advancing the approach and enhancing the consistency. Res Synth Methods 2014;5:371-85.

37. The Joanna Briggs Institute. Methodology for JBI Scoping reviews: Joanna Briggs Insitute, 2015.

38. Peters MD, Godfrey CM, Khalil H, et al. Guidance for conducting systematic scoping reviews. Int J Evid Based Healthc 2015;13:141-6.

39. U.S. National Library of Medicine. Fact Sheet @ MEDLINE, PubMed, and PMC (PubMed Central): How are they different? 2017. https:// www.nlm.nih.gov/pubs/factsheets/dif_med_pub.html (accessed 20 May 2017).

40. Landis JR, Koch GG. The measurement of observer agreement for categorical data. Biometrics 1977;33:159-74.

41. Barr H, Freeth D, Hammick M, et al. Evaluations of interprofessional education. London, 2000. 